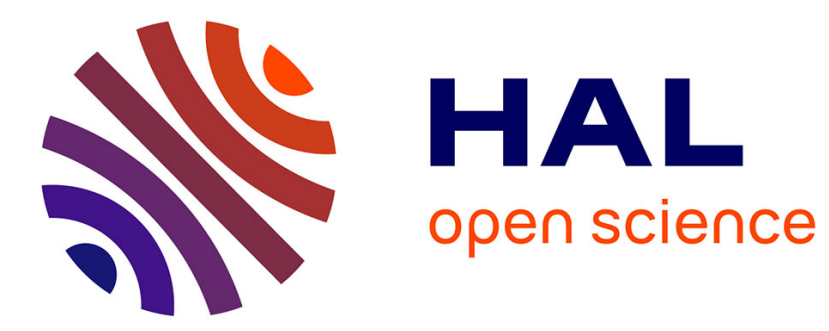

\title{
Comprendre, choisir et acheter des LEDs UV
}

Walter Fiedler, Christian Merry, Peter E. Gordon

\section{To cite this version:}

Walter Fiedler, Christian Merry, Peter E. Gordon. Comprendre, choisir et acheter des LEDs UV.

Photoniques, 2021, 106, pp.48-52. 10.1051/photon/202110648 . hal-03150627

\section{HAL Id: hal-03150627 \\ https://hal.science/hal-03150627}

Submitted on 23 Feb 2021

HAL is a multi-disciplinary open access archive for the deposit and dissemination of scientific research documents, whether they are published or not. The documents may come from teaching and research institutions in France or abroad, or from public or private research centers.
L'archive ouverte pluridisciplinaire HAL, est destinée au dépôt et à la diffusion de documents scientifiques de niveau recherche, publiés ou non, émanant des établissements d'enseignement et de recherche français ou étrangers, des laboratoires publics ou privés. 


\section{COMPRENDRE, CHOISIR ET ACHETER DES LEDS UV}

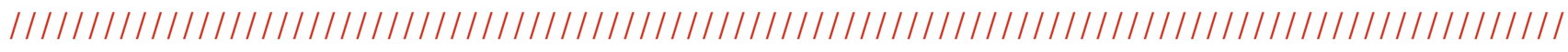

\section{Walter FIEDLER ${ }^{1}$, Christian MERRY' ${ }^{1}$, Peter E. GORDON ${ }^{2}$}

${ }^{1}$ Laser Components, 45 bis route des gardes, 92190 Meudon, France

${ }^{2}$ Bolb Inc., 52 Wright Brothers Ave, Livermore CA 94551, USA

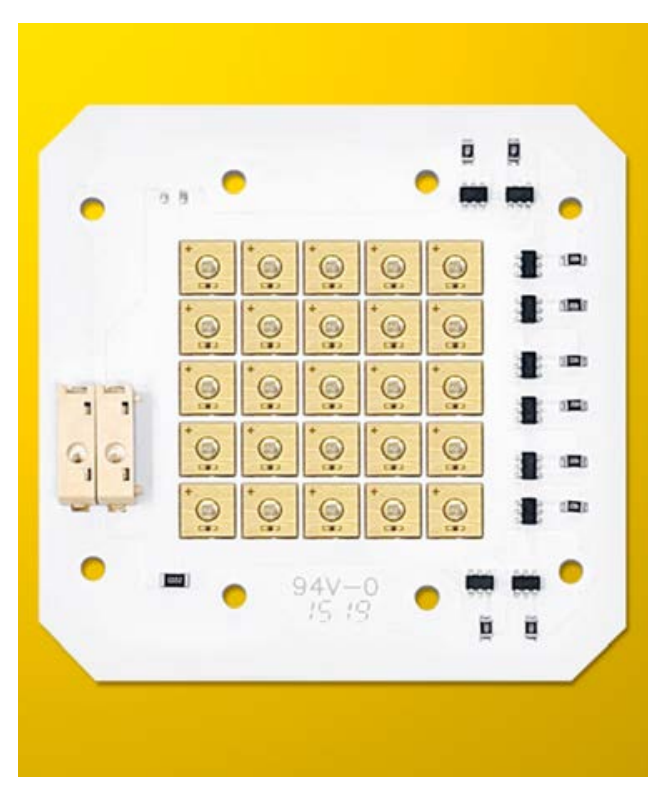

\section{RENDRE L'OPAQUE}

TRANSPARENT

Jusqu'à présent, la croissance du marché des LED UVC a été entravée par un problème technique majeur : le matériau semi-conducteur. Les LED de plus grande longueur d'onde - en particulier le vert, le bleu et les UVA - utilisent principalement des composés de GaN comme matériaux semi-conducteurs, car ils offrent la large bande interdite nécessaire à cette partie du spectre. Cette technologie est bien développée et utilisée pour un large éventail d'applications. L'AlGaN, qui est nécessaire pour les longueurs d'onde UVC, ne présente qu'un rendement électrique-optique décrit souvent sous le terme Wall Plug Efficiency (WPE) de 3 à $5 \%$, ce qui

En 1992, le prix Nobel Japonais Isamu Akasaki a mis au point la première diode électroluminescente (DEL) pour le spectre ultraviolet. Depuis lors, cette technologie relativement jeune $n$ 'a cessé de conquérir de nouveaux domaines d'application. Il est difficile de suivre l'évolution actuelle du marché, en particulier dans les courtes longueurs d'onde UV entre $280 \mathrm{~nm}$ et $100 \mathrm{~nm}$, tant le développement technique progresse rapidement. Nous proposons ici un aperçu et des aides à la décision pratiques dans la recherche d'une LED UV adaptée à l'application visée.

\section{https://doi.org/10.1051/photon/202110648}

signifie que seule une petite partie de la puissance électrique appliquée est transformée en puissance optique. La plupart des LED développées jusqu'à présent n'ont pas le rendement nécessaire pour concurrencer sérieusement les technologies existantes telles que les lampes à mercure. Ce n'est que récemment que de nouvelles technologies ont permis de résoudre ce problème et d'augmenter considérablement le rendement.

\section{PERCÉE TECHNOLOGIQUE GRÂCE AU GAZ À TROUS BIDIMENSIONNELS}

La nature profonde inhérente aux accepteurs des semi-conducteurs à large bande interdite rend difficile la formation de contacts $\mathrm{p}$-ohmiques et l'alimentation des trous, ce qui empêche une transformation suffisante de l'électricité en lumière. La société BOLB a mis au point une méthode pour augmenter le nombre de trous disponibles et, par conséquent, augmenter les performances des LED. Au lieu d'un semi-conducteur massif à large bande interdite, ils ont utilisé une combinaison ultra-mince d'une couche d'AlN dopée au Mg et d'une couche de p-AlGaN/AlGaN (voir Fig. 1). En raison de la grande différence entre les constantes intrinsèques du réseau des couches respectives, l'AlN subit une grande contrainte de traction biaxiale s'élevant à $0,94 \%$ sans relaxation. En raison de la polarisation piézoélectrique, une densité de charge 
de $-1.28 \times 10^{13} \mathrm{~cm}^{-2}$ est créée à la surface de cette couche. La fine couche de GaN dopée au Mg sous la couche de p-AlN est utilisée pour maximiser la discontinuité de polarisation spontanée entre l'AlN et le GaN, ce qui crée une densité de charge nette de $-3.25 \times 10^{13} \mathrm{~cm}^{-2}$.

La combinaison des charges piézoélectriques et spontanées transforme la couche de p-AlN en un condensateur virtuel à plaques parallèles avec une densité de charge d'environ $-4.53 \times 10^{13} \mathrm{~cm}^{-2}$. Elle produit un champ électrique de près de $9,0 \mathrm{MV} /$ $\mathrm{cm}$, qui laisse passer à travers le matériau des trous précédemment liés à des accepteurs dans un tunnel de p-AlN avec une probabilité de 0,65 $\%$. À une concentration d'accepteurs de $2,0 \times 10^{20} \mathrm{~cm}^{-3}$, cet effet entraîne une concentration de trous de 1.3 $\times 10^{18} \mathrm{~cm}^{-3}$. La plupart d'entre eux sont entraînés vers la surface de la couche ultra-mince d'AlN où ils forment ce qu'on appelle un gaz à trous bidimensionnels (2DHG), qui rend le matériau AlN habituellement isolant pratiquement conducteur.

Bolb a appliqué cette technologie pour créer des LED UVC présentant une tension directe typique de $6,5 \mathrm{~V}$ à $271 \mathrm{~nm}$. Avec un WPE de $8,55 \%$, elles atteignent un flux radiant de $100 \mathrm{~mW}$. Leurs LED germicides

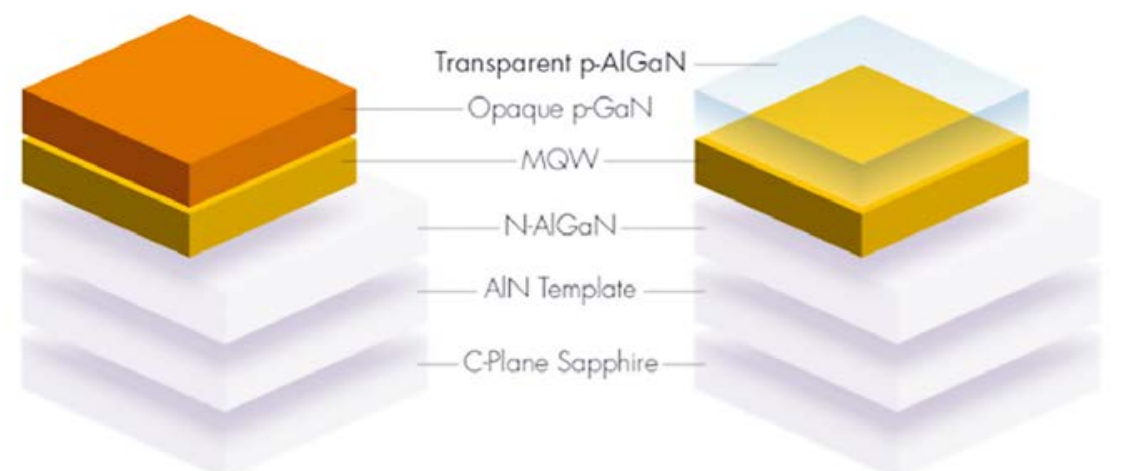

Traditional Design:

Non-Transparent $\mathrm{P}$ layer

BOLB epi:

Transparent $P$ layer and

Efficient Hole Injector

Figure 1.

Schéma de LEDs en AIGaN pour la génération de rayonnement UVC. Gauche : architecture conventionnelle. Droite: procédé développé par BOLB remplaçant la couche opaque de GaN par une couche p transparente d'AlGaN. En combinaison avec un injecteur de trous efficace, cette approche permet d'obtenir des puissances de sortie UVC élevées.

sont parmi les plus puissantes actuellement sur le marché. Sur la base de leur technologie, Bolb s'attend à une augmentation considérable du WPE au cours de la prochaine décennie, avec pour objectif de dépasser les $50 \%$ d'ici 2030.

\section{CRISTAUX À COUCHES}

HAUTEMENT RÉFLÉCHISSANTES

RIKEN et Panasonic ont suivi une voie différente plus conventionnelle, en concevant de nouvelles structures de couches et de nouvelles techniques de croissance cristalline pour l'AlN et l'AlGaN à large bande interdite. Leur objectif principal était d'améliorer l'efficacité de l'extraction de la lumière (Light extraction efficien cy - LEE), c'est-à-dire la fraction des photons générés qui s'échappent de la LED. Pour atteindre cet objectif, ils ont associé une couche transparente de contact $\mathrm{p}$ à une électrode hautement réfléchissante. La première consistait en une couche de $\mathrm{p}$-AlGaN avec une composition de 50 à $70 \%$ d'Al,

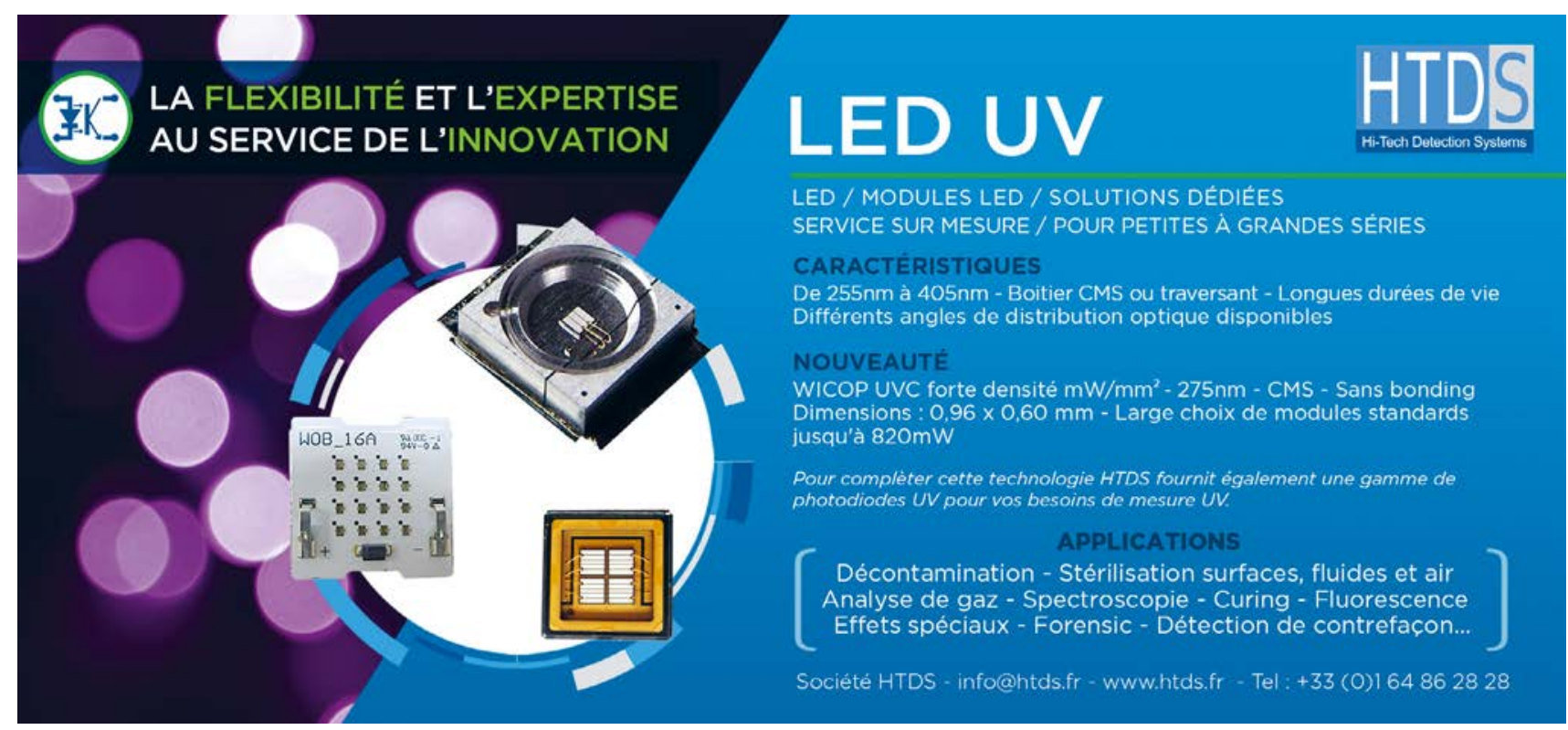


mais la faible concentration de trous de ce matériau a conduit à une tension de fonctionnement considérablement plus élevée que celle utilisée dans les LED classiques.

Pour maintenir la tension au niveau habituel, les scientifiques ont utilisé la nano-impression et la gravure sèche par plasma inductif pour créer un cristal photonique bidimensionnel à faible dommage et hautement réfléchissant, qui a été appliqué sur la surface de la couche de contact supérieure en p-GaN. Cette nouvelle conception de diode a été complétée par deux types d'électrodes de type $\mathrm{p}$ - à savoir une électrode Ni à faible réflexion (30\%) et une électrode $\mathrm{Ni} / \mathrm{Mg}$ à forte réflexion (80\%). Grâce à ces mesures, ils ont pu créer une diode UVC-LED AlGaN avec une efficacité quantique externe (EQE) d'environ $20 \%$.

\section{UNE QUESTION \\ DE LONGUEUR D'ONDE}

Dans la recherche de « la bonne » LED UV, l'application - et donc la longueur d'onde souhaitée - est bien sûr une priorité absolue. Le rayonnement ultraviolet est la gamme de longueurs d'onde comprise entre le spectre visible et les rayons X (c'est-à-dire les faisceaux de haute énergie à ondes courtes entre environ $400 \mathrm{~nm}$ et $100 \mathrm{~nm}$ ). Tout comme la lumière visible peut être divisée en différentes couleurs, le rayonnement UV invisible est traditionnellement divisé en différentes gammes de longueurs d'onde. Il a été démontré que cette catégorisation peut également s'appliquer à l'utilisation industrielle et commerciale de la « lumière UV ».

\section{UV-A}

L'UV-A décrit la gamme de longueurs d'onde entre la fin de la lumière visible (environ $400 \mathrm{~nm}$ ) et $320 \mathrm{~nm}$. Dans la gamme de longueurs d'onde comprise entre $420 \mathrm{~nm}$ et $390 \mathrm{~nm}$, les premières LED ont été disponibles vers la fin des années 1990. Cette lumière dite «noire » sert principalement à exciter la fluorescence et est utilisée, par exemple, dans la fabrication des billets de banque, des cartes d'identité et des documents à l'épreuve de la contrefaçon. Toutes ces applications ne nécessitent que de faibles niveaux de puissance de sortie.

Le rayonnement UV entre $390 \mathrm{~nm}$ et $350 \mathrm{~nm}$ est utilisé dans l'industrie pour la polymérisation des adhésifs, des revêtements, des encres et de nombreux autres matériaux. En raison de leur grande efficacité, de leur faible coût de fonctionnement et de leur conception compacte, les diodes remplacent de plus en plus d'autres technologies, comme les lampes à vapeur de mercure.

\section{UV-B}

UV-B est le terme utilisé pour les longueurs d'onde comprises entre $320 \mathrm{~nm}$ et $280 \mathrm{~nm}$. Les diodes électroluminescentes polyvalentes entre $340 \mathrm{~nm}$ et $300 \mathrm{~nm}$ sont la dernière introduction sur le marché dans le segment des longueurs d'onde UVA/B. Elles offrent un grand potentiel pour de nombreuses applications différentes, telles que le séchage UV, l'analyse de l'ADN, la dermatologie et la technologie des capteurs.

\section{UV-C}

L'UV-C commence à $280 \mathrm{~nm}$ et se termine à $200 \mathrm{~nm}$. Comme la lumière UV-C est complètement absorbée par l'atmosphère, aucune des créatures vivantes sur Terre n'a développé de défenses naturelles contre ce

Figure 2 .

Tente de Décontamination SARS-CoV-2, UVC, Wuhan, mars 2020.
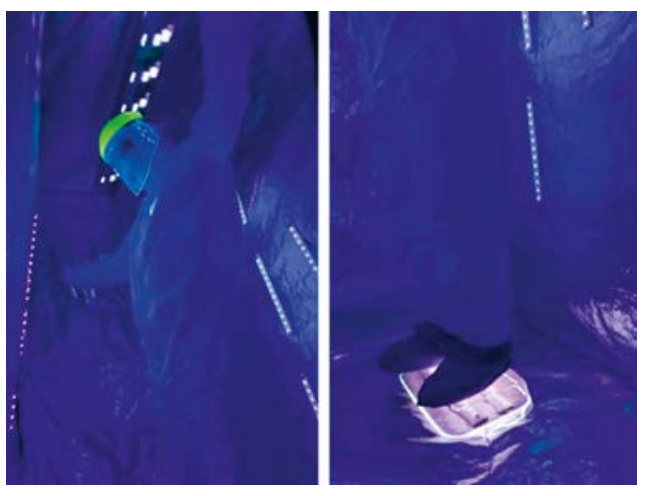

rayonnement de haute énergie. Cela s'applique aux humains, ainsi qu'aux bactéries et aux virus. Le rayonnement est absorbé par les brins d'ADN et d'ARN des organismes conduisant à une « agglutination » des séquences de nucléotides qui détruit l'information génétique d'une cellule. Ainsi, la cellule ne peut plus se reproduire et peut même être tuée.

Les LED germicides à $270 \mathrm{~nm}$ sont très proches du maximum d'absorption des acides nucléiques $(265 \mathrm{~nm})$ et peuvent donc être utilisées de manière particulièrement ciblée dans la lutte contre les micro-organismes (voir Fig. 2). Outre la longueur d'onde et la puissance de sortie, les propriétés spécifiques des différents germes doivent également être prises en compte dans les applications de désinfection. Un taux de désinfection suffisant de 99,9999\% n'est souvent atteint qu'après une longue période d'irradiation.

\section{PLUS PETIT ET PLUS EFFICACE QUE LES SOLUTIONS PRÉCÉDENTES}

La longueur d'onde émise et la puissance d'une LED jouent toutes deux un rôle crucial dans son succès commercial. Les LED UV-C à ondes courtes ont fait des progrès significatifs ces dernières années. Les lampes à vapeur de mercure, qui sont utilisées comme sources de rayonnement depuis des décennies, sont de plus en plus remplacées par des LED car elles présentent plusieurs avantages par rapport à cette technologie traditionnelle:

- Alors que les lampes à vapeur de mercure nécessitent des tensions élevées et génèrent beaucoup de chaleur, les LED peuvent fonctionner avec des tensions et des courants continus faibles. Cela rend également possible une utilisation mobile.

- Comme les LED n'émettent pratiquement pas de rayonnement thermique dans la direction du faisceau, elles peuvent également être utilisées pour le traitement de matériaux sensibles à la chaleur.

- Contrairement aux lampes à vapeur de mercure, les LED ne nécessitent 
pas de «temps de chauffe » et sont immédiatement prêtes à l'emploi. Cela permet également de faire fonctionner les émetteurs avec des impulsions de l'ordre de la milliseconde et de la nanoseconde.

- La longueur d'onde, l'intensité du rayonnement et la forme du faisceau peuvent être ajustées relativement facilement pendant la production. Alors que les lampes à vapeur de mercure n'émettent que certaines longueurs d'onde, les LED peuvent être fabriquées pour une large gamme de longueurs d'onde en variant la composition des matériaux semi-conducteurs. L'intensité du faisceau peut être facilement contrôlée par l'alimentation électrique.

- Les LED sont petites, compactes et peuvent être intégrées dans presque toutes les applications. Contrairement aux lampes à vapeur de mercure, elles ne sont pas fragiles et ne contiennent pas de substances toxiques.

\section{DES MARCHÉS SATURÉS ET UN POTENTIEL \\ DE CROISSANCE ELEVÉ}

La situation du marché des LED UV dépend fortement de la longueur d'onde. L'état de développement technique est crucial car le cycle d'adaptation technologique s'applique également dans ce domaine. Il commence par une innovation technique qui ouvre un nouveau marché : les quantités sont encore faibles, et les prix sont élevés. Au fil du temps, la nouvelle technologie s'établit sur le marché. Les quantités augmentent à mesure que la demande augmente et les prix baissent en conséquence. Le résultat est un marché saturé avec une production industrielle de masse, des quantités élevées et des prix bas. Les différents marchés des LED UV se trouvent à des stades différents de ce cycle de développement. La règle empirique est la suivante : "plus la longueur d'onde est courte, plus la LED est chère ».

La technologie des LED à lumière noire est mature depuis des décennies. Les coûts de fabrication sont par conséquent faibles, et les produits sont proposés à bas prix par de nombreux fabricants. La pression d'innover dans la gamme de longueurs d'onde entre $390 \mathrm{~nm}$ et $350 \mathrm{~nm}$ a entraîné une croissance rapide de ce segment de marché ces dernières années. Les coûts de fabrication sont toujours nettement plus élevés que dans le segment de la lumière noire ; toutefois, les méthodes de production de plus en plus efficaces et les volumes croissants entraînent une baisse continue des prix.

Dans le segment des UVB, il a été possible, ces dernières années, d'augmenter la production des différentes diodes électroluminescentes; cependant, la valeur de l'efficacité quantique est très faible.

Le marché des applications de désinfection est actuellement le segment le plus passionnant et le plus dynamique. Il est actuellement encore dominé par les anciennes lampes à vapeur de mercure ; toutefois, ces lampes sont encombrantes et fragiles. Elles ne sont donc pas adaptées aux applications mobiles. Ce n'est qu'il y a seulement quelques années que les LED UV-C ont également été développées avec un niveau de performance qui rend possible des solutions de désinfection efficaces. Les analystes s'attendent donc à ce que le marché des LED UV-C se développe rapidement au cours des prochaines années.

\section{L'INNOVATION TECHNIQUE DÉCLENCHE DE NOUVEAUX STIMULI DE CROISSANCE}

Maintenant que les bases techniques ont été posées, il est possible pour la première fois de faire des prévisions quant au développement futur du marché. Les experts l'assimilent au

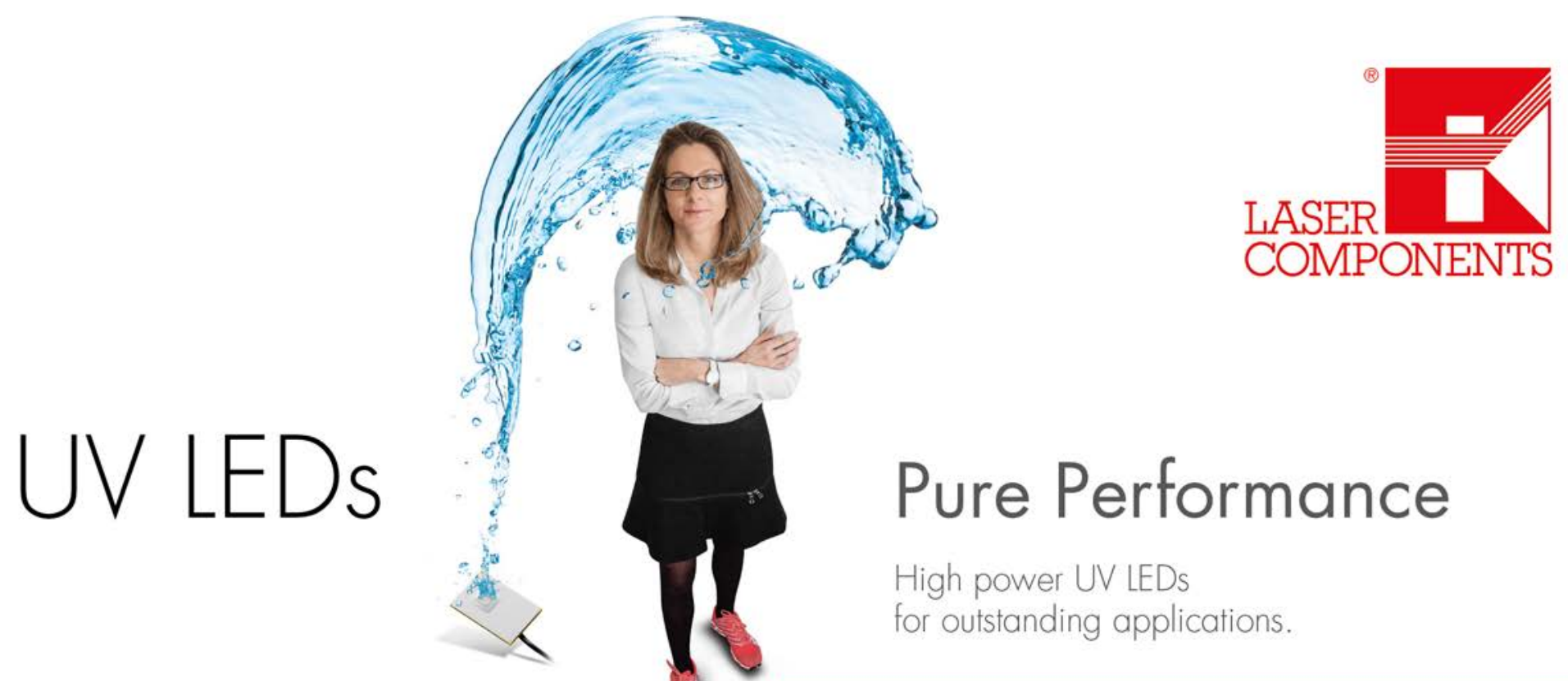


marché des LED bleues, qui a été créé à la fin des années 80 avec une percée technique similaire. En outre, la crise actuelle du corona donne une impulsion durable au marché. Même avant la COVID-19, les analystes prédisaient que le marché des LED UV dépasserait la barre du milliard de dollars en quelques années seulement. Des géants du marché tels que Nishia ou San'an investissent actuellement des sommes importantes dans les LED UV-C, car ils attendent des marges élevées de ce segment en pleine croissance. Dans le même temps, de petites start-ups innovantes s'efforcent d'accroitre leur avance technologique pour influencer le développement futur du marché.

Des puissances de $400 \mathrm{~mW}$ avec des rendements quantiques de $20 \%$ sont attendus d'ici quelques années. À ce rythme, le rendement en photons pourrait atteindre plus de $50 \%$ d'ici 2030. Le prix serait alors d'environ un dollar par watt optique. Compte tenu des nombreux avantages décrits ci-dessus des LED par rapport aux lampes à vapeur de mercure, il est probable que les LED commencent à évincer du marché les solutions établies beaucoup plus tôt, d'autant plus que de nouveaux segments de marché qui ne peuvent être couverts par les technologies classiques (par exemple, dans le traitement mobile de l'eau) s'ouvrent en même temps.
La sensibilisation accrue à l'hygiène et à la désinfection résultant de l'actuelle pandémie de corona va encore stimuler la demande et le développement technique. Plusieurs grands constructeurs automobiles ont déjà annoncé qu'ils investiraient des millions dans des solutions de désinfection par UV pour l'intérieur des véhicules, qui ne peuvent être mises en œuvre qu'avec des LED appropriées. Des applications similaires sont actuellement en cours de développement pour les transports publics et les transports de patients.

\section{RÉFÉRENCE}

[1] B. Seme, I. Streit, Photonics Views 16, 34-37 (2019)

\begin{tabular}{|c|c|c|c|c|c|}
\hline SOCIÉTÉ & TYPE DE DISTRIBUTION & FABRICANT & TYPE DE LED & PUISSANCE MAX & SITES WEB \\
\hline APTECH/RUTRONIK & $\begin{array}{l}\text { Société basée } \\
\text { en Allemagne }\end{array}$ & Lextar & $\begin{array}{l}\text { UVC (270nm) } \\
\text { UVA(395-405nm) }\end{array}$ & $\begin{array}{l}20 \mathrm{~mW} \\
570-600 \mathrm{~mW}\end{array}$ & www.aptechsc.com \\
\hline ES France & $\begin{array}{l}\text { Société basée en France/ } \\
\text { site marchand }\end{array}$ & Refond & LED : UV-B \& UV-C & $12 \mathrm{~mW}$ & www.es-france.com \\
\hline HTDS & Société basée en France & Seoul Viosys & $\begin{array}{l}\text { UVC }(275 \mathrm{~nm}) \\
\text { UVB }(285-310 \mathrm{~nm}) \\
\text { UVA }(340-420 \mathrm{~nm}) \\
\text { Module } 4 \text { LEDs UVC }(275 \mathrm{~nm})\end{array}$ & $\begin{array}{l}50 \mathrm{~mW} \\
10 \mathrm{~mW} \\
5300 \mathrm{~mW} \\
280 \mathrm{~mW} \text { (info } \\
\text { Tarif } 70 € \text { ) }\end{array}$ & www.htds.fr \\
\hline LASER2000 & Société basée en France & Crystal IS & UVC(255-285nm) & $3 \mathrm{~mW}$ & $\begin{array}{l}\text { https://register. } \\
\text { gotowebinar.com/ } \\
\text { register/119356795396350220 }\end{array}$ \\
\hline LASER COMPONENTS SAS & Société basée en France & BOLB & $\begin{array}{l}\text { UVC }(272 \mathrm{~nm}) \\
\text { Module } 4 \text { LEDs UVC }(272 \mathrm{~nm}) \\
\text { Module } 12 \text { LEDs UVC }(272 \mathrm{~nm}) \\
\text { Module } 25 \text { LEDs UVC }(272 \mathrm{~nm})\end{array}$ & $\begin{array}{l}100 \mathrm{~mW} \\
360 \mathrm{~mW} \\
1,2 \mathrm{~W} \\
2 \mathrm{~W}\end{array}$ & www.lasercomponents.fr \\
\hline LUMITRONIX & $\begin{array}{l}\text { Site marchand/Site } \\
\text { marchand/ Société basée } \\
\text { en Italie }\end{array}$ & Nichia & $\begin{array}{l}\text { UVC }(280 n m) \\
\text { UVA }(365-405 n m)\end{array}$ & $\begin{array}{l}76 \mathrm{~mW} \\
4640 \mathrm{~mW}\end{array}$ & https://b2b.lumitronix.com \\
\hline Mouser & Site marchand & Luminus & $\begin{array}{l}\text { UVC(275-286nm) } \\
\text { UVA(365-405nm) }\end{array}$ & $\begin{array}{l}80 \mathrm{~mW} \\
80 \mathrm{~mW}\end{array}$ & www.luminus.com \\
\hline $\begin{array}{l}\text { Mouser/Rutronik/PAN } \\
\text { ELEKTRON S.R.L }\end{array}$ & Sites marchands & LiteOn & $\begin{array}{l}\text { UVC(270-285nm) } \\
\text { UVA(365-430nm) }\end{array}$ & $\begin{array}{l}80 \mathrm{~mW} \\
1,8 \mathrm{~W}\end{array}$ & $\begin{array}{l}\text { https://optoelectronics. } \\
\text { liteon.com }\end{array}$ \\
\hline Rutronik & Site marchand & Stanley & UVC $(265-275 \mathrm{~nm})$ & $50 \mathrm{~mW}$ & $\begin{array}{l}\text { www.stanley-components. } \\
\text { com/en }\end{array}$ \\
\hline Rutronik/Avnet/Mouser & Site marchand & Vishay & $\begin{array}{l}\text { UVC }(270-285 \mathrm{~nm}) \\
\text { UVB(360-400nm) }\end{array}$ & $\begin{array}{l}19 \mathrm{~mW} \\
1325 \mathrm{~mW}\end{array}$ & www.vishay.com \\
\hline \multirow{3}{*}{ Pas de distributeur identifié } & & PhotonWave & $\begin{array}{l}\text { UVC }\left(255 \mathrm{~nm}^{-265} \mathrm{~nm} \& 275 \mathrm{~nm}\right) \\
\text { UVB }(308 \mathrm{~nm})\end{array}$ & $\begin{array}{l}42 \mathrm{~mW} \\
40 \mathrm{~mW}\end{array}$ & www.iuva.org \\
\hline & & SanAn & $\begin{array}{l}\text { UVC }(270-280 \mathrm{~nm}) \\
\text { Module UVC }(275-286 \mathrm{~nm}) \\
\text { UVB }(305-315 \mathrm{~nm})\end{array}$ & $\begin{array}{l}65 \mathrm{~mW} \\
200 \mathrm{~mW} \\
6 \mathrm{~mW}\end{array}$ & www.sanan-e.com/en \\
\hline & & Jason & UVC (260-270nm) & $1 \mathrm{~mW}$ & www.qdjason.com/en \\
\hline
\end{tabular}

